\title{
Baked Food Consumption Is A Risk Factor for Chronic Kidney Disease: A 1:1 Paired Case-control Study
}

\section{Xiaoying Lai}

Southern Medical University Nanfang Hospital

Shiyu Zhou

Southern Medical University

Zewei Lin

Tencent

\section{Chuanjie Yan}

Southern Medical University

Hong Zhu

Southern Medical University

\section{Wenyuan Li}

Southern Medical University Nanfang Hospital

Shengli An ( $\sim$ asl0418@126.com )

southern medical university

\section{Ping Ouyang}

Southern Medical University Nanfang Hospital

\section{Research article}

Keywords: lifestyle behavior, chronic kidney disease, case-control study

Posted Date: May 22nd, 2020

DOI: https://doi.org/10.21203/rs.3.rs-30191/v1

License: (c) (i) This work is licensed under a Creative Commons Attribution 4.0 International License. Read Full License 


\section{Abstract \\ Background}

Chronic Kidney Disease (CKD) has become a global public health problem. Identifying the risk factors of CKD can provide strategies for the prevention of CKD. Studies showed that lifestyles play an important role in CKD, but the relationship between them remained unclear. Thus we aimed to explore the association of lifestyle behaviors (the dietary habits especially) with CKD.

\section{Methods}

A 1:1 matched case-control study including 1414 participates from the HUIQIAO health database system from Jan. 2015 to Dec. 2018 was conducted. Our main outcome measure was the diagnose of CKD, and exposures were lifestyle behaviors measured by a questionnaire. The anthropometric characteristics were included as confounding variables.

\section{Results}

We used conditional logistics regression to assess the odds ratios (ORs) and adjusted ORs for the risk factors. With the assumption of missing at random (MAR) pattern, the missing values of confounding variables and exposures were handled by multiple imputation. We found that lifestyle behaviors regarding skipping breakfast $\geq 3$ times per week $(1.672,95 \% \mathrm{Cl}, 1.086-2.574)$, sleep efficiency $\leq 75 \%(1.633,95 \%$ $\mathrm{Cl}, 1.195-2.232)$, consuming baked food frequently $(1.683,95 \% \mathrm{Cl}, 1.163-2.434)$, proper intake of oil $(0.789,95 \% \mathrm{Cl}, 0.624-0.996)$, proper intake of aquatic product $(0.732,95 \% \mathrm{Cl}, 0.567-0.944)$, proper intake of soybean and nuts $(0.625,95 \% \mathrm{Cl}, 0.447-0.873)$ were associated with CKD.

\section{Conclusion}

These results suggest that moderate intake of soybean, nuts, and aquatic product, control oil intake, having breakfast at least 4 times per week, maintaining good sleep efficiency, and consuming less baked foods may contribute to prevent the development of CKD.

\section{Trial registration:}

This study is registered at http://www.chictr.org.cn (ChiCTR2000031864).

\section{Introduction}


Chronic kidney disease (CKD) was an occult and common disease which had a 1 in 7 to 10 adult prevalence all over the world[1]. CKD was a direct reason for cardiovascular disease and lead to many complications, such as chronic kidney disease-mineral and bone disorder (CKD-MBD)[2], end-stage kidney disease (ESKD)[3, 4]. In 2017, there were 697.5 million CKD patients worldwide and almost a third of them lived-in China and India[3]. However, the economic and social load of CKD as well as the potential risk factors remained understudied in many areas of the world. So far, it was widely accepted that the traditional risk factors for CKD were high body mass index, impaired fasting plasma glucose, high blood pressure, unbalanced diet such as high sodium consumption, and lead[5]. Most of the risk factors were able to be adjusted by lifestyle behaviors intervention. On one hand, the impact of lifestyle habits on health was cumulative; on the other hand, there were many aspects of lifestyle habits, both positive and negative, which often influenced each other[6] in the human body's metabolism process. Further exploration and research to evaluate the quality of lifestyle habits are needed. Until our study, only a few articles had searched out the association between the effects of lifestyle behaviors and the prevalence of CKD. For example, a meta-analysis inferred that keeping a healthy diet, such as DASH diet and MD diet, was associated with CKD prevention and decreased the risk of death caused by renal cause at the same time[7]. While an unhealthy diet pattern might lead to increasing the risk of CKD reported by a crosssectional study called Irish Nun and Eye Study[8]. A review about the association between obstructive sleep apnea (OSA) and CKD suggested that sleep-related breathing disorder, such as OSA, indirectly induce CKD by the hypertension, endothelial dysfunction and oxidative[9]. Thus, we hypothesize that interference of lifestyle behaviors such as sleep behaviors, lifestyle behaviors including diet patterns may be an effective approach to reducing CKD risk. Besides, more potential and unconventional risk factors should be identified due to the CKD's ethnic disparities[10].

Therefore, this study aims to evaluate the association between the unhealthy lifestyle behaviors and the risk of CKD in ordinary Chinese.

\section{Material And Methods Study population}

This 1:1 matched case-control study retrieved data from the HUIQIAO health database system provided by Nanfang Hospital, Southern Medical University from Jan. 2015 to Dec. 2018. Demographic and clinical variables included age, gender, weight, systolic blood pressure (SBP), diastolic blood pressure (DBP), waist circumference (WC), fasting blood glucose (FBG), serum creatinine (SCr), serum uric acid (SUA), total cholesterol (CHOL), triglycerides (TG), low-density lipoprotein (LDL-C), high-density lipoprotein (HDL-C), very low-density lipoprotein (VLDL-C) and body mass index (BMI). Lifestyle variables included smoking status, drinking consumption, dietary intakes (oil, cereals, fruits, vegetables, poultry and meat, aquatic product, egg, milk and dairy products, soybean and nuts), habitual history (frequency of breakfast or night snack per week, frequency of consuming carbonated drinks, juice, coffee, tea, offal, salted food, smoked food, barbecue or baked food), sleep habits (sleep efficiency), physical activity, and sedentary. Briefly, 707 cases aged 18-88 years entered this study. 
The cases included subjects meet the CKD diagnosis. Participants in the control group, from the same system, were individually matched by age ( \pm 2 years) and sex. Both controls and cases were subject to the same inclusion and exclusion criteria as cases except for a history of CKD. Individuals who rejected or incompletely finished the self-reported lifestyle questionnaires during the interview $(n=300$ for cases, $n=$ 16978 for controls) and age below 18 years $(n=220)$ were excluded. For people who had health checkups more than once, only included the first time. The study procedure was recapped in Fig. 1.

\section{Measures}

CKD was defined as eGFR $<60 \mathrm{~mL} / \mathrm{min} / 1.73 \mathrm{~m}^{2}$, proteinuria positive $[+,++$ or +++$]$, or both[11]. The calculated formula of eGFR was developed by Andrew S. Levey[12] in 2009 as below:

$\mathrm{eGFR}_{\mathrm{CKD}-\mathrm{EPI}}\left(\mathrm{mL} / \mathrm{min} / 1.73 \mathrm{~m}^{2}\right)=141 \times \min (S c r / \kappa, 1)^{a_{\times}} \max (S c r / \kappa, 1)^{-1.209} \times 0.993^{\text {age }} \times 1.018($ female $)$

Where for male, $\mathrm{k}$ was 0.9 and a was -0.411 , while for female, $\mathrm{k}$ was 0.7 and a was -0.329 , min indicates the minimum of $\mathrm{Scr} / \mathrm{K}$ or 1 , and max indicates the maximum of $\mathrm{Scr} / \mathrm{K}$ or 1 . To cover $\mathrm{SCr}$ from $\mathrm{mg} / \mathrm{dl}$ to umol/L, multiply by 88.4. The unit of age was the year; the unit of eGFR was $\mathrm{ml} / \mathrm{min} / 173 \mathrm{~m}^{2}$. The eGFR was a more accurate measure for renal function than $\mathrm{SCr}$ and identified patients with mild renal impairment despite normal or nearly normal SCr levels[13]. Moreover, the eGFR was a strong predictor of cardiovascular events. Urinary albumin was measured with immunoturbidimetric tests from a morning spot urine sample and the urine test results were classified as $[-, \pm,+,++$ or +++$]$.

As for anthropometric measures, used a computer body scale to recorded weight and height when the subjects minimally clothed, without shoes, and the shoulders were in normal alignment. The computer body scale needed an accuracy of up to 100 and a minimum measurement of $1 \mathrm{~mm}$. The BMl calculation formula was: BMI $\left(\mathrm{kg} / \mathrm{m}^{2}\right)=$ weight $(\mathrm{kg}) /[\text { height }(\mathrm{m})]^{2}$. Take WC measurements by a tape measure at the level of the umbilicus without any pressure on the abdomen. And the measurements were recorded to the nearest $0.1 \mathrm{~cm}$. The classifications of BMI and WC referred previous studies[14, 15]. Blood pressure was measured in the right arm, after resting for sitting at least 15 minutes and using the average of duplicate measurements. A blood sample was taken after $12 \mathrm{~h}$ of overnight fasting according to the standard protocol and centrifuged within 30-45 min of collection. All of the blood analyses were performed at the Nanfang hospital laboratory on the day of blood collection. The diagnosis of metabolic syndrome refers to the 2005 IDF standard[16]. Dyslipidemia was defined as meeting one or more of the following conditions: TC $\geq 5.2 \mathrm{mmol} / \mathrm{L} ; \mathrm{TG} \geq 1.7 \mathrm{mmol} / \mathrm{L} ; \mathrm{LDL}-\mathrm{C} \geq 3.4 \mathrm{mmol} / \mathrm{L} ; \mathrm{HDL}-\mathrm{C} \otimes 1.0 \mathrm{mmol} / \mathrm{L}[17]$. Diagnosis of hyperuricemia: SUA $\geq 420 \mathrm{mmol} / \mathrm{L}$ for male, $\geq 360 \mathrm{mmol} / \mathrm{L}$ for female[18].

Trained doctors or research staff interviewed all participants with a structured questionnaire containing demographic and lifestyle-related characteristics. A food-frequency questionnaire was used to collect dietary intake information, providing an instruction manual that included photographs of general foods and portion sizes. Food consumption frequency was estimated at per day, per week, per month, per year or never. The classifications of dietary intakes referred to the Dietary Guidelines for Chinese Residents 


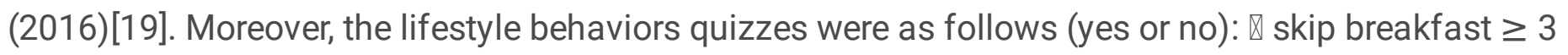
times per week; $₫$ night snack $\geq 3$ times per week; $\Downarrow$ frequently or every day of consuming carbonated drinks, juice, coffee, tea, offal, salted food, smoked food, barbecue and baked food. For drinking, data were available by drinking or not, the frequency of drinking (per day/per week), years of drinking, type, and milliliters of drinking each time. Alcohol consumptions were combined into 1 measure of average daily consumption (grams of pure alcohol). Based on the levels of alcohol consumption, subjects were classified into moderates alcohol consumption ( $<15 \mathrm{~g} / \mathrm{d}$ for female and $<25 \mathrm{~g} / \mathrm{d}$ for male) and high alcohol consumption ( $\geq 15 \mathrm{~g} / \mathrm{d}$ for female and $\geq 25 \mathrm{~g} / \mathrm{d}$ for male). Individuals who had smoked more than 6 months before the interview were classified as current smokers; otherwise, subjects were classified as non-smokers (including ever smoked but quit for more than 2 years). Physical activity[20] was categorized in low, moderate, and high, based on the physical activity questionnaire. Furthermore, sitting for more than 8 hours per day were classified as sedentary. Sleep habits were collected as follows: "Did you have snore or partners had told you that you had snore while sleeping?", " When you go to bed?", "When you get up in the morning?", "Please write down your actual sleep time per night (not bedtime)". The calculation formula for sleep efficiency was:

sleepefficiency $=[($ actual sleep time $) /($ time to get up - time to sleep $)] \times 100 \%$

\section{Statistics analysis}

Anthropometric, clinical, and dietary intake results were shown in Tables 1 and 2. After matching, a total of 707 paired subjects were selected for the final analysis. Clinical and lifestyle characteristics of participants with cases and controls were summarized as mean (SD), median (first quartile, third quartile) or number (\%) as appropriate. Baseline characteristics were analyzed using the t-test, ANOVA, and Chisquare test as appropriate. Conditional logistic regression was used to assess the association between CKD and lifestyle behaviors. Univariable and multivariable analyses were applied to calculate odds ratios (ORs) with their corresponding 95\% confidence intervals (Cls). Covariates included in the multivariable analyses were weight, BMI, WC, DBP, SBP, TG, HDL-C, VLDL-C, SUA, FBG, hyperuricemia, metabolic syndrome, WC groups and dyslipidemia, whose $P$ values were less than 0.2 in univariable analysis. With the assumption of missing at random (MAR), missing data were handled via the predictive-meanmatching method (PMM)[21] of multiple imputation (MI). This method was repeated 5 times to acquire five complete datasets, which reduced the bias in a data set by means of imputation by taking real values sample from the data and reflected the uncertainty for missing data. Using R version 3.6.2 and SPSS 22.0 software. to perform statistical analysis. $P<0.05$ was considered statistically significant. 
Table 1

Demographics and Clinical Characteristics of Study Population in the Case-control Study $(\mathrm{n}=1,414)$

\begin{tabular}{|lllll}
\hline Variables & Control $(\mathbf{n}=\mathbf{7 0 7})$ & Case $(\mathbf{n}=\mathbf{7 0 7})$ & $\mathrm{t} / \mathrm{Z} / \mathbf{x}^{2}$ & P-value \\
\hline Weight, kg & $69.4(62.4,75.9)$ & $70.9(62.9,78.9)$ & -3.676 & $<0.001$ \\
\hline WC, $\mathrm{cm}$ & $85.36(9.43)$ & $88.33(10.54)$ & 6.047 & $<0.001$ \\
\hline SBP, mmHg & $124(113,135)$ & $130(117,143)$ & -5.824 & $<0.001$ \\
\hline DBP, mmHg & $73(66,81)$ & $77(68,87)$ & -5.335 & $<0.001$ \\
\hline CHOL, mmol/L & $5.34(1.09)$ & $5.34(1.3)$ & 0.059 & 0.953 \\
\hline LDL-C, mmol/L & $3.43(0.83)$ & $3.38(0.89)$ & -1.081 & 0.280 \\
\hline TG, mmol/L & $1.34(0.97,1.87)$ & $1.51(1.08,2.27)$ & -4.868 & $<0.001$ \\
\hline HDL-C, mmol/L & $1.2(1.04,1.37)$ & $1.13(0.97,1.32)$ & -4.660 & $<0.001$ \\
\hline VLDL-C, mmol/L & $0.62(0.42,0.86)$ & $0.68(0.46,0.91)$ & -3.135 & 0.002 \\
\hline FBG, mmol/L & $5.2(4.86,5.64)$ & $5.46(4.99,6.37)$ & -7.218 & $<0.001$ \\
\hline SUA, $\mu$ mol/L & $402.79(89.46)$ & $441.21(102.51)$ & 7.633 & $<0.001$ \\
\hline Serum creatinine, $\mu$ mol/L & $77(69,87)$ & $87(72,110)$ & -11.184 & $<0.001$ \\
\hline BMI, kg/m 2 & $24.69(3.17)$ & $25.5(3.59)$ & 4.643 & $<0.001$ \\
\hline History of T2DM & $50(7.07)$ & $92(13.01)$ & 13.81 & $<0.001$ \\
\hline History of Hypertension & $115(16.27)$ & $199(28.15)$ & 28.89 & $<0.001$ \\
\hline Metabolic syndrome & $133(18.81)$ & $280(39.60)$ & 73.91 & $<0.001$ \\
\hline Dyslipidemia & $522(73.83)$ & $551(77.93)$ & 3.25 & 0.077 \\
\hline Hyperuricemia & $303(42.86)$ & $406(57.43)$ & 30.01 & $<0.001$ \\
\hline BMl groups & & & 32.50 & $<0.001$ \\
\hline Light weight & $328(46.39)$ & $282(39.89)$ & & \\
\hline Normal weight & & & & \\
\hline Overweight & & $13(1.84)$ & & \\
\hline
\end{tabular}

WC: Waist circumference; SBP: Systolic blood pressure; DBP: Diastolic blood pressure; CHOL: Total cholesterol; LDL-C: Low-Density Lipoprotein Cholesterol; TG: Triglycerides; HDL-C: High-Density Lipoprotein Cholesterol; VLDL-C: Very Low-Density Lipoprotein Cholesterol; FBG: Fasting Blood Glucose; SUA: Serum uric acid; BMI: Body mass index; T2DM:Type 2 diabetes mellitus.

Note: Boldface indicates statistical significance $(P$-value $<0.2)$. Continuous variables: mean (SD), median (with interquartile range); categorical variables: number (with percentage); categorical variables: number (with percentage). 


\begin{tabular}{|c|c|c|c|c|}
\hline Variables & Control $(n=707)$ & Case $(n=707)$ & $t / Z / x^{2}$ & $P$-value \\
\hline Obesity & $85(12.02)$ & $167(23.62)$ & & \\
\hline WC groups & & & 39.00 & $<0.001$ \\
\hline Normal & $347(49.08)$ & $275(38.9)$ & & \\
\hline Overweight & $273(38.61)$ & $256(36.21)$ & & \\
\hline Obesity & $87(12.31)$ & $176(24.89)$ & & \\
\hline \multicolumn{5}{|c|}{$\begin{array}{l}\text { WC: Waist circumference; SBP: Systolic blood pressure; DBP: Diastolic blood pressure; CHOL: Total } \\
\text { cholesterol; LDL-C: Low-Density Lipoprotein Cholesterol; TG: Triglycerides; HDL-C: High-Density } \\
\text { Lipoprotein Cholesterol; VLDL-C: Very Low-Density Lipoprotein Cholesterol; FBG: Fasting Blood } \\
\text { Glucose; SUA: Serum uric acid; BMI: Body mass index; T2DM:Type } 2 \text { diabetes mellitus. }\end{array}$} \\
\hline \multicolumn{5}{|c|}{$\begin{array}{l}\text { Note: Boldface indicates statistical significance }(P \text {-value }<0.2) \text {. Continuous variables: mean }(S D) \text {, } \\
\text { median (with interquartile range); categorical variables: number (with percentage); categorical } \\
\text { variables: number (with percentage). }\end{array}$} \\
\hline
\end{tabular}


Table 2

Lifestyle Characteristics of Study Population in the Case-control Study $(n=1,414)$

\begin{tabular}{|c|c|c|c|}
\hline Variables & Control $(n=707)$ & Case $(n=707)$ & $P$-value \\
\hline \multicolumn{4}{|l|}{ Unhealthy } \\
\hline Lack of physical activity & $314(44.41 \%)$ & $332(46.96 \%)$ & 0.337 \\
\hline Sedentary $>8$ h per day & $99(14.00 \%)$ & $85(12.02 \%)$ & 0.268 \\
\hline Skip breakfast $\geq 3$ times per week & $54(7.64 \%)$ & $85(12.02 \%)$ & 0.006 \\
\hline Night snack $\geq 3$ times per week & $45(6.36 \%)$ & $61(8.63 \%)$ & 0.106 \\
\hline Eat offal frequently & $40(5.66 \%)$ & $45(6.36 \%)$ & 0.576 \\
\hline Eat salted food frequently & $56(7.92 \%)$ & $60(8.49 \%)$ & 0.698 \\
\hline Eat smoked food frequently & $24(3.39 \%)$ & $27(3.82 \%)$ & 0.669 \\
\hline Eat barbecue frequently & $43(6.08 \%)$ & $45(6.36 \%)$ & 0.134 \\
\hline Eat baked food frequently & $83(11.74 \%)$ & $102(14.43 \%)$ & 0.134 \\
\hline Drink juice frequently & $30(4.24 \%)$ & $45(6.36 \%)$ & 0.075 \\
\hline Drink coffee frequently & $52(7.36 \%)$ & $57(8.06 \%)$ & 0.618 \\
\hline Drink tea frequently & $508(71.85 \%)$ & $538(76.10 \%)$ & 0.069 \\
\hline Drink carbonated drinks frequently & $24(3.39 \%)$ & $37(5.23 \%)$ & 0.089 \\
\hline Sleep efficiency $\leq 75 \%$ & $95(13.44 \%)$ & $131(18.53 \%)$ & 0.009 \\
\hline Current smoker & $242(34.23 \%)$ & $289(40.88 \%)$ & 0.010 \\
\hline Excessive drinking & 79(11.17\%) & $112(15.84 \%)$ & 0.010 \\
\hline \multicolumn{4}{|l|}{ Healthy } \\
\hline Proper intake of Oil(25-30 g/day) & $444(62.8 \%)$ & $404(57.14 \%)$ & 0.030 \\
\hline Proper intake of cereals $(250-400 \mathrm{~g} /$ day $)$ & $402(56.86 \%)$ & $380(53.75 \%)$ & 0.239 \\
\hline Proper intake of fruits $(250-300 \mathrm{~g} /$ day $)$ & $339(47.95 \%)$ & $333(47.10 \%)$ & 0.749 \\
\hline Proper intake of vegetables(300-500 g/day) & $401(56.72 \%)$ & $405(57.28 \%)$ & 0.830 \\
\hline Proper intake of poultry and meat( $40-75 \mathrm{~g} /$ day $)$ & $192(27.16 \%)$ & $184(26.03 \%)$ & 0.630 \\
\hline Proper intake of aquatic product( $40-75 \mathrm{~g} /$ day $)$ & $216(30.55 \%)$ & $172(24.33 \%)$ & 0.009 \\
\hline
\end{tabular}

Note: Boldface indicates statistical significance $(P$-value $<0.05)$. 


\begin{tabular}{|llll|}
\hline Variables & Control $(\mathbf{n}=707)$ & Case $(\mathbf{n}=707)$ & $P$-value \\
\hline Proper intake of egg(40-50 g/day) & $227(32.11 \%)$ & $226(31.97 \%)$ & 0.955 \\
\hline Proper intake of milk and dairy products(300 g/day) & $50(7.07 \%)$ & $40(5.66 \%)$ & 0.276 \\
\hline Proper intake of soybean and nuts $(25-30 \mathrm{~g} /$ day $)$ & $116(16.41 \%)$ & $78(11.03 \%)$ & 0.003 \\
\hline Note: Boldface indicates statistical significance $(P$-value $<0.05)$. & & \\
\hline
\end{tabular}

\section{Results}

\section{Subjects selection}

Figure 1 showed the process of selection of the eligible subjects. There were 43865 cases in the medical examination system from Jan. 2015 to Dec. 2018. Subjects aged below 18 years were excluded $(n=220)$. Only the medical examination results for the first time were included for individuals who came to the hospital for more than once during the study period $(n=9452)$.

The case group included 1031 patients who had CKD, as determined by eGFR ( $\left.<60 \mathrm{ml} / \mathrm{min} / 1.73 \mathrm{~m}^{2}\right)$ and urine protein $(+/++/+++)$. Before matching, we excluded those who did not finish the lifestyle questionnaires. The other participants without CKD were matched 1:1 in age ( \pm 2 years), sex with the control group (Fig. 1).

Table 1 demonstrated the characteristics of CKD and non-CKD groups. The status of health conditions between the two groups was significantly different, especially metabolic syndrome (39.6\% vs. $18.81 \%$ ), hyperuricemia ( $57.43 \%$ vs. $42.86 \%$ ), obesity (BMI: $23.62 \%$ vs. $12.02 \%$; WC: $24.89 \%$ vs. $12.31 \%$ ), and dyslipidemia (77.93\% vs. $73.83 \%)$.

Compared with controls, cases had a higher frequency of the lifestyle behaviors regarding skipping breakfast $\geq 3$ times a week ( $7.64 \%$ vs. $12.02 \%$ ), sleep efficiency $\leq 75 \%$ ( $13.44 \%$ vs. $18.53 \%)$, smoking (34.23\% vs. $40.88 \%)$, and excessive drinking (11.17\% vs. $15.48 \%$ ) and lower frequency of the lifestyle behaviors like proper intake of oil $(62.80 \%$ vs. $57.14 \%)$, proper intake of the aquatic product $(30.55 \%$ vs. $24.33 \%)$, proper intake of soybean and nuts ( $16.41 \%$ vs. $11.03 \%$ ) (Table 2 ).

Table 3 and Fig. 2 showed the results of conditional logistic regression. In model 1, proper intake of oil, proper intake of aquatic products, proper intake of soybean and nuts, and sleep efficiency $\leq 75 \%$ were associated with CKD. After adjusting potential confounding variables, skip breakfast $\geq 3$ times per week $(1.672,95 \% \mathrm{Cl}, 1.086-2.574, P=0.002)$, sleep efficiency $\leq 75 \%(1.633,95 \% \mathrm{Cl}, 1.195-2.232, P=0.002)$, eat baked food frequently $(1.683,95 \% \mathrm{Cl}, 1.163-2.434, P=0.006)$, proper intake of oil $(0.789,95 \% \mathrm{Cl}$, $0.624-0.996, P=0.047)$, proper intake of aquatic products $(0.732,95 \% \mathrm{Cl}, 0.567-0.944, P=0.016)$, and proper intake of soybean and nuts $(0.625,95 \% \mathrm{Cl}, 0.447-0.873, P=0.006)$ were associated with CKD. 
Table 3

ORs of CKD and Corresponding 95\% Cls According to different variables $(n=1,414)$

\begin{tabular}{|c|c|c|c|c|c|c|}
\hline \multirow{2}{*}{ Theaspectsoflifestylebehaviors ${ }^{a}$} & \multicolumn{3}{|c|}{ Model 1} & \multicolumn{3}{|c|}{ Model 2} \\
\hline & OR & $95 \% \mathrm{Cl}$ & $\mathbf{P}$ & OR & $95 \% \mathrm{Cl}$ & $\mathbf{P}$ \\
\hline Lack of physical activity & 1.062 & $\begin{array}{l}0.852 \\
1.324\end{array}$ & 0.590 & 1.132 & $\begin{array}{l}0.897 \\
1.429\end{array}$ & 0.296 \\
\hline Sedentary $>8 \mathrm{~h}$ per day & 0.781 & $\begin{array}{l}0.563 \\
1.082\end{array}$ & 0.137 & 0.771 & $\begin{array}{l}0.546 \\
1.089\end{array}$ & 0.139 \\
\hline Skip breakfast $\geq 3$ times per week & 1.515 & $\begin{array}{l}1.005 \\
2.283\end{array}$ & 0.047 & 1.672 & $\begin{array}{l}1.086 \\
2.574\end{array}$ & 0.020 \\
\hline Night snack $\geq 3$ times per week & 0.971 & $\begin{array}{l}0.613 \\
1.538\end{array}$ & 0.899 & 0.937 & $\begin{array}{l}0.571 \\
1.537\end{array}$ & 0.795 \\
\hline Eat offal frequently & 1.045 & $\begin{array}{l}0.641 \\
1.703\end{array}$ & 0.860 & 0.953 & $\begin{array}{l}0.570 \\
1.593\end{array}$ & 0.853 \\
\hline Eat salted food frequently & 0.918 & $\begin{array}{l}0.601 \\
1.401\end{array}$ & 0.690 & 0.942 & $\begin{array}{l}0.605 \\
1.468\end{array}$ & 0.793 \\
\hline Eat smoked food frequently & 0.987 & $\begin{array}{l}0.524 \\
1.861\end{array}$ & 0.968 & 0.792 & $\begin{array}{l}0.403 \\
1.557\end{array}$ & 0.499 \\
\hline Eat barbecue frequently & 0.902 & $\begin{array}{l}0.552 \\
1.473\end{array}$ & 0.680 & 0.938 & $\begin{array}{l}0.558 \\
1.578\end{array}$ & 0.809 \\
\hline Eat baked food frequently & 1.379 & $\begin{array}{l}0.972 \\
1.956\end{array}$ & 0.072 & 1.683 & $\begin{array}{l}1.163 \\
2.434\end{array}$ & 0.006 \\
\hline Drink juice frequently & 1.430 & $\begin{array}{l}0.804 \\
2.541\end{array}$ & 0.223 & 1.283 & $\begin{array}{l}0.699 \\
2.354\end{array}$ & 0.422 \\
\hline Drink coffee frequently & 0.906 & $\begin{array}{l}0.589 \\
1.394\end{array}$ & 0.653 & 0.943 & $\begin{array}{l}0.598 \\
1.487\end{array}$ & 0.801 \\
\hline Drink tea frequently & 1.202 & $\begin{array}{l}0.938 \\
1.539\end{array}$ & 0.146 & 1.083 & $\begin{array}{l}0.834 \\
1.408\end{array}$ & 0.550 \\
\hline Drink carbonated drinks frequently & 1.145 & $\begin{array}{l}0.616 \\
2.126\end{array}$ & 0.669 & 1.308 & $\begin{array}{l}0.683 \\
2.505\end{array}$ & 0.418 \\
\hline
\end{tabular}

BMI: Body mass index; WC: Waist circumference; DBP: Diastolic blood pressure; SBP: Systolic blood pressure; TG: Triglycerides; HDL-C: High-Density Lipoprotein Cholesterol; VLDL-C: Very Low-Density Lipoprotein Cholesterol; SUA: Serum uric acid; FBG: Fasting Blood Glucose.

Note: Boldface indicates statistical significance $(P$-value $<0.05)$. Model 1: adjustment for age, sex. Model 2: adjustment for age, sex, weight, BMI, WC, DBP, SBP, TG, HDL-C, VLDL-C, SUA, FBG, hyperuricemia, metabolic syndrome, WC groups.

a. All these factors were binary variables $(1=$ Yes. $0=\mathrm{No})$ that denoted whether participants have that lifestyle behavior or not and 0 as a reference. 


\begin{tabular}{|c|c|c|c|c|c|c|}
\hline \multirow{2}{*}{ Theaspectsoflifestylebehaviors ${ }^{a}$} & \multicolumn{3}{|c|}{ Model 1} & \multicolumn{3}{|c|}{ Model 2} \\
\hline & OR & $95 \% \mathrm{Cl}$ & $\mathbf{P}$ & OR & $95 \% \mathrm{Cl}$ & $\mathbf{P}$ \\
\hline Sleep efficiency $\leq 75 \%$ & 1.504 & $\begin{array}{l}1.119 \\
2.023\end{array}$ & 0.007 & 1.633 & $\begin{array}{l}1.195 \\
2.232\end{array}$ & 0.002 \\
\hline Current smoker & 1.209 & $\begin{array}{l}0.957 \\
1.527\end{array}$ & 0.111 & 1.096 & $\begin{array}{l}0.854 \\
1.405\end{array}$ & 0.471 \\
\hline Excessive drinking & 1.334 & $\begin{array}{l}0.963 \\
1.847\end{array}$ & 0.083 & 1.084 & $\begin{array}{l}0.766 \\
1.534\end{array}$ & 0.650 \\
\hline Proper intake of Oil(25-30 g/day) & 0.779 & $\begin{array}{l}0.624 \\
0.971\end{array}$ & 0.027 & 0.789 & $\begin{array}{l}0.624 \\
0.996\end{array}$ & 0.047 \\
\hline Proper intake of cereals(250-400 g/day) & 0.881 & $\begin{array}{l}0.710 \\
1.094\end{array}$ & 0.252 & 0.877 & $\begin{array}{l}0.698 \\
1.102\end{array}$ & 0.259 \\
\hline Proper intake of fruits $(250-300 \mathrm{~g} /$ day $)$ & 1.016 & $\begin{array}{l}0.816 \\
1.264\end{array}$ & 0.888 & 1.019 & $\begin{array}{l}0.809 \\
1.284\end{array}$ & 0.872 \\
\hline $\begin{array}{l}\text { Proper intake of vegetables( } 300- \\
500 \mathrm{~g} / \text { day })\end{array}$ & 1.061 & $\begin{array}{l}0.853 \\
1.320\end{array}$ & 0.595 & 1.059 & $\begin{array}{l}0.841 \\
1.333\end{array}$ & 0.626 \\
\hline $\begin{array}{l}\text { Proper intake of poultry and meat(40- } \\
75 \mathrm{~g} / \text { day })\end{array}$ & 0.982 & $\begin{array}{l}0.768 \\
1.256\end{array}$ & 0.886 & 1.040 & $\begin{array}{l}0.803 \\
1.347\end{array}$ & 0.767 \\
\hline $\begin{array}{l}\text { Proper intake of aquatic product(40- } \\
75 \mathrm{~g} / \text { day) }\end{array}$ & 0.737 & $\begin{array}{l}0.579 \\
0.938\end{array}$ & 0.013 & 0.732 & $\begin{array}{l}0.567 \\
0.944\end{array}$ & 0.016 \\
\hline Proper intake of egg $(40-50 \mathrm{~g} /$ day $)$ & 1.052 & $\begin{array}{l}0.831 \\
1.332\end{array}$ & 0.673 & 1.068 & $\begin{array}{l}0.833 \\
1.370\end{array}$ & 0.604 \\
\hline $\begin{array}{l}\text { Proper intake of milk and dairy } \\
\text { products }(300 \mathrm{~g} / \text { day })\end{array}$ & 0.782 & $\begin{array}{l}0.500 \\
1.224\end{array}$ & 0.282 & 0.797 & $\begin{array}{l}0.492 \\
1.293\end{array}$ & 0.358 \\
\hline $\begin{array}{l}\text { Proper intake of soybean and nuts(25- } \\
30 \mathrm{~g} / \text { day })\end{array}$ & 0.610 & $\begin{array}{l}0.445 \\
0.836\end{array}$ & 0.002 & 0.625 & $\begin{array}{l}0.447 \\
0.873\end{array}$ & 0.006 \\
\hline
\end{tabular}

BMI: Body mass index; WC: Waist circumference; DBP: Diastolic blood pressure; SBP: Systolic blood pressure; TG: Triglycerides; HDL-C: High-Density Lipoprotein Cholesterol; VLDL-C: Very Low-Density Lipoprotein Cholesterol; SUA: Serum uric acid; FBG: Fasting Blood Glucose.

Note: Boldface indicates statistical significance $(P$-value $<0.05)$. Model 1: adjustment for age, sex. Model 2: adjustment for age, sex, weight, BMI, WC, DBP, SBP, TG, HDL-C, VLDL-C, SUA, FBG, hyperuricemia, metabolic syndrome, WC groups.

a. All these factors were binary variables $(1=$ Yes. $0=\mathrm{No})$ that denoted whether participants have that lifestyle behavior or not and 0 as a reference.

We used MI to handle the missing data with five imputed data sets, and we reported a comparison between the multiple imputation analysis (Table 3) and the complete case (Table 4). After adjusting confounding variables, aquatic product was found to be statistically significant in multiple imputation analysis but not in complete case analysis $(0.916,95 \% \mathrm{Cl}, 0.658-1.274, P=0.601)$. In addition to that, 
other results from complete case analysis were consistent with multiple imputation analysis. Table 3 ORs of CKD and Corresponding 95\% Cls According to different variables Among 707 Cases and 707 Controls. 


\section{Theaspectsoflifestylebehaviors ${ }^{a}$}

OR $\quad 95 \% \mathrm{Cl} \quad P$-value

Lack of physical activity

Sedentary $>8$ h per day

Skip breakfast $\geq 3$ times per week

Night snack $\geq 3$ times per week

Eat offal frequently

Eat salted food frequently

Eat smoked food frequently

Eat barbecue frequently

Eat baked food frequently

Drink juice frequently

Drink coffee frequently

Drink tea frequently

Drink carbonated drinks frequently

Sleep efficiency $\leq 75 \%$

Current smoker

Excessive drinking

Proper intake of Oil(25-30 g/day)

Proper intake of cereals(250-400 g/day)

Proper intake of fruits $(250-300 \mathrm{~g} /$ day $)$

Proper intake of vegetables(300-500 g/day)

Proper intake of poultry and meat(40-75 g/day)
1.034

0.720

1.855

1.063

0.821

0.851

0.860

0.811

1.829

0.925

1.071

1.052

1.742

1.681

1.214

0.842

0.940

1.091

1.001

1.174

1.124
0.831

0.175

$0.448,1.157$

0.024

$1.084,3.175$

$0.594,1.902$

0.837

$0.426,1.581 \quad 0.555$

$0.485,1.493$

0.573

$0.361,2.052 \quad 0.735$

$0.424,1.552$

0.527

$1.122,2.981 \quad 0.015$

$0.422,2.026 \quad 0.845$

$0.611,1.878 \quad 0.810$

$0.745,1.485$

0.774

$0.769,3.944 \quad 0.183$

$1.135,2.489$

0.010

$0.884,1.667$

0.231

$0.547,1.296 \quad 0.434$

$0.692,1.276$

0.690

$0.811,1.469 \quad 0.564$

$0.741,1.353 \quad 0.994$

$0.870,1.585$

0.293

$0.802,1.574$

0.497

BMI: Body mass index; WC: Waist circumference; DBP: Diastolic blood pressure; SBP: Systolic blood pressure; TG: Triglycerides; HDL-C: High-Density Lipoprotein Cholesterol; VLDL-C: Very Low-Density Lipoprotein Cholesterol; SUA: Serum uric acid; FBG: Fasting Blood Glucose.

Note: Boldface indicates statistical significance $(P<0.05)$. Adjustment for age, sex, weight, $\mathrm{BMI}, \mathrm{WC}$, DBP, SBP, TG, HDL-C, VLDL-C, SUA, FBG, hyperuricemia, metabolic syndrome, WC groups.

a. All these factors were binary variables $(1=$ Yes. $0=\mathrm{No})$ that denoted whether participants have that lifestyle behavior or not and 0 as a reference. 


\section{Theaspectsoflifestylebehaviors ${ }^{a}$ \\ OR \\ $95 \% \mathrm{Cl}$ \\ P-value}

\begin{tabular}{llll} 
Proper intake of aquatic product $(40-75 \mathrm{~g} /$ day $)$ & 0.916 & $0.658,1.274$ & 0.601 \\
\hline Proper intake of egg(40-50 g/day) & 0.904 & $0.651,1.254$ & 0.544 \\
\hline Proper intake of milk and dairy products $(300 \mathrm{~g} /$ day $)$ & 0.765 & $0.391,1.500$ & 0.436 \\
\hline Proper intake of soybean and nuts $(25-30 \mathrm{~g} /$ day $)$ & 0.590 & $0.391,0.889$ & 0.012
\end{tabular}

BMI: Body mass index; WC: Waist circumference; DBP: Diastolic blood pressure; SBP: Systolic blood pressure; TG: Triglycerides; HDL-C: High-Density Lipoprotein Cholesterol; VLDL-C: Very Low-Density Lipoprotein Cholesterol; SUA: Serum uric acid; FBG: Fasting Blood Glucose.

Note: Boldface indicates statistical significance $(P<0.05)$. Adjustment for age, sex, weight, $\mathrm{BMI}, \mathrm{WC}$, DBP, SBP, TG, HDL-C, VLDL-C, SUA, FBG, hyperuricemia, metabolic syndrome, WC groups.

a. All these factors were binary variables $(1=$ Yes. $0=\mathrm{No})$ that denoted whether participants have that lifestyle behavior or not and 0 as a reference.

\section{Discussion}

In our study, we first revealed the relationship between CKD in 1414 health check-up populations aged 18-88 years from Guangdong, China with lifestyle behaviors by the use of a 1:1 paired case-control study. The major finding of this study was that lifestyle behaviors including skipping breakfast $\geq$ three times per week, consuming baked food frequently, sleep efficiency $\leq 75 \%$ were risk factors for the development of CKD, while proper consumption of oil, aquatic product, soybean, and nuts contributed to prevent CKD. Furthermore, after adjusting the traditional risk factor, those lifestyle behaviors were still found to be associated with the incidence of CKD. The results underscored the importance of lifestyle behaviors in the management of CKD. Supporting evidence revealed that lifestyle behaviors had complicated associations with the risks of hypertension[22], CVD (stroke and heart failure)[23], metabolic syndrome[24], cancer[25], T2DM[26], and CKD with T2DM[27]. As we all know, diabetes, hypertension and obesity are important traditional risk factors for CKD[28]. To develop targeted prevention strategies, it is important to investigate the nontraditional risk factors of CKD, such as lifestyle-related factors, which are likely modifiers of CKD risk. In a cross-sectional study enrolling 25,493 middle-aged participants, those with unhealthy lifestyles were more likely to have proteinuria[29]. Ryoma Michishita et al found that changing from a healthy to an unhealthy lifestyle could significantly increase the incidence of CKD[30]. Conversely, keeping healthy lifestyle behaviors, such as habitual moderate exercise and no bedtime snacking, was important to reduce the risk of CKD[31].

In our investigation, baked foods were first observed to be associated with CKD. In cases, the proportion of consuming baked food frequently was 1.683 times higher than that in controls. The specific mechanisms underlying the baked foods on the prevalence of CKD were unclear. One possibility was that high 18:2 trans fatty acids which were abundant in baked products were associated with increased risk of nonfatal myocardial infarction[32]. Typically, CVD including nonfatal myocardial infarction was strongly 
associated with the development of CKD[33]. Furthermore, the intermediate products of the Maillard reaction and caramelization, such as dicarbonyl compounds, which possibility increased the total body AGEs load[34] which might lead to diabetes, obesity and renal failure[34, 35]. We also hypothesized that the acrylamide (ACR), commonly detected in the baked foods, was a risk factor for CKD. After exposure, ACR renal tubular cells undergo vacuolar degenerative changes, inflammatory cell infiltration, and periglomerular edema[36]. The nephrotoxicity of ACR increased serum urea, creatinine, uric acid, and renal proinflammatory cytokine levels, while also inducing lipid peroxidation and DNA damage[37]. Hence, we concluded that the increasing consumption of baked foods might impair renal function and promote the development of CKD.

To our investigation, the moderate consumption of the aquatic product was protective factors for preventing CKD. Admittedly, it is controversial whether aquatic product intake takes a positive effect on health or not. Fernanda Santin et al defined an "unhealthy" pattern including fish intake based on exploratory factor analysis in a cross-section study and concluded that "unhealthy" patterns lead to diminished renal function and developed CKD[38]. While in a cohort study enrolling 4133 healthy individuals aged 18-30 years, Inwhee Park et al found that the intake of LC $\omega-3 P U F A$, which mainly provided by fish, was inversely associated with the development of CKD[39].

Additionally, kipping breakfast was a risk factor for CKD, which was in line with previous studies[31]. Then, individuals with moderate consumption of soybean and nuts were at a lower risk of CKD. Similar conclusion was found in a prospective cohort study including 15,792 white and black adults from four U.S. communities[40]. Individuals with CKD were more likely to have a lower sleep efficiency. In a metaanalysis, Xiu HongYang et al confirmed that short sleep duration or sleep efficiency $\leq 75 \%$ were associated with increased mortality in CKD patients[41]. In contrast, among African Americans, sleep quality could be improved by improving sleep hygiene behaviors[42]. As for cooking oil, different kinds of cooking oil had different influences on diseases including renal function. In an animal model stereological study, the authors revealed that $20 \%$ sesame oil might lead to renal deformities[43]. However, saturated FAts in animal oil caused insulin resistance which contributes to the development of CKD[44]. Interestingly, in our work, the moderate consumption of cooking oil (25-30 ml/d) had a positive effect on the CKD prevention regardless of the type of cooking oil.

Several potential limitations should also be considered. First, collecting of lifestyle information based on self-reported questionnaires and retrospective data analysis were possibilities of information bias and recall bias. To reduce bias, trained researchers would relieve participants during progresses finishing the questionnaire. Graphic explanations next to the titles and reference photos with a standardized portion size for food consumption provided for individuals. Second, the data were incomplete for some individuals, which may result in misclassification of diagnosis. As the missing rate was lower than $12 \%$ and the original data set was large enough, using the predictive mean matching method to impute the data. Third, it had the potential for residual confounders from unmeasured demographics data such as educational attainment, marriage status and monthly household income. 
The large sample size provided an opportunity to adjust for a large range of confounding. On one hand, some participants might have health checkups more than once because of the analysis including data from 2015 to 2018; on the other hand, they would change their lifestyle behaviors for keeping health after health examination. Only included the first biochemical test result, as well as CKD diagnosis. Especially, adult dietary habits remained stable[45]. In this way, we could avoid the possibility of reverse cause and reduce the consistency between the cases and controls.

\section{Conclusion}

In conclusion, lifestyle behaviors were associated with the prevalence of CKD. To develop targeted health management strategies, we suggest that healthy populations should take proper dietary patterns, especially oil, aquatic products, soybean and nuts. Also, it is essential to have breakfast at least 4 times a week, take fewer baked food and keep a better sleep efficiency. Future studies of CKD prevention should conduct to interacting the lifestyle behaviors.

\section{Abbreviations}

CKD: chronic kidney disease; eGFR:estimated glomerular filtration rate; WC:Waist circumference; BMI:body mass index; SBP:Systolic blood pressure; DBP:Diastolic blood pressure; CHOL:Total cholesterol; LDL-C:Low-Density Lipoprotein Cholesterol; TG:Triglycerides; HDL-C:High-Density Lipoprotein Cholesterol; VLDL-C:Very Low-Density Lipoprotein Cholesterol; FBG:Fasting Blood Glucose; SUA:Serum uric acid; SCr:serum creatinine; T2DM:Type 2 diabetes mellitus; CKD-MBD:chronic kidney disease-mineral and bone disorder; ESKD:end-stage kidney disease; OSA:obstructive sleep apnea; ACR:acrylamide; SD:standard deviation; OR:odds ratio; Cl:confidence interval; MAR:missing at random; PMM:predictive-mean-matching method; Ml:multiple imputation.

\section{Declarations}

\section{Acknowledgements}

Not applicable.

\section{Ethics approval and consent to participate}

This study conformed to the Helsinki Declaration, and approved by the Ethics Committee of Nanfang Hospital, Southern Medical University (NFEC-2019-058).

\section{Funding}


This research was supported by the Clinical Research Program of Nanfang Hospital, Southern Medical University [grant numbers 2018CR009].

The content is solely the responsibility of the authors and does not necessarily represent the official views of Nanfang Hospital, Southern Medical University. The funder had no role in the design and conduct of the study; collection, management, analysis, and interpretation of the data; preparation, review, or approval of the manuscript; or the decision to submit the manuscript for publication.

\section{Consent for publication}

Not applicable

\section{Availability of data and materials}

Data sharing is not applicable to this article as no datasets were generated or analysed during the current study.

\section{Author contribution}

Xiaoying Lai, Shiyu Zhou and Zewei Lin are co-first authors. Shengli An and Ping Ouyang are cocorrespondence authors.

Xiaoying Lai, Shengli An and Ping Ouyang conceptualized and designed the study. Shiyu Zhou and Zewei Lin carried out the statistical analysis; Shiyu Zhou interpreted the data; Xiaoying Lai obtained funding and drafted the manuscript; Chuanjie Yan had full access to all the data in the study; Shengli An and Ping Ouyang reviewed and revised the manuscript. All the authors critically revised the manuscript for important intellectual content and approved the final manuscript as submitted and agreed to be accountable for all aspects of the work.

\section{Competing interests}

The authors have no financial relationships relevant to this article to disclose. All authors have no conflicts of interest relevant to this article to disclose.

\section{References}

1. Tuttle KR, Alicic RZ, Duru OK, Jones CR, Daratha KB, Nicholas SB, McPherson SM, Neumiller JJ, Bell DS, Mangione CM, et al. Clinical Characteristics of and Risk Factors for Chronic Kidney Disease Among Adults and Children: An Analysis of the CURE-CKD Registry. JAMA Netw Open. 2019;2(12):e1918169. 
2. Wang AY, Akizawa T, Bavanandan S, Hamano T, Liew A, Lu KC, Lumlertgul D, Oh KH, Zhao MH, KaShun Fung S, et al: 2017 Kidney Disease: Improving Global Outcomes (KDIGO) Chronic Kidney Disease-Mineral and Bone Disorder (CKD-MBD) Guideline Update Implementation: Asia Summit Conference Report. Kidney Int Rep 2019, 4(11):1523-1537.

3. Webster AC, Nagler EV, Morton RL, Masson P. Chronic Kidney Disease. Lancet. 2017;389(10075):1238-52.

4. Levin A, Stevens PE, Bilous RW, Coresh J, Winearls CG. Kidney disease: Improving global outcomes (KDIGO) CKD work group. KDIGO 2012 clinical practice guideline for the evaluation and management of chronic kidney disease. Kidney International Supplements. 2013;3(1):1-150.

5. Collaboration GBDCKD. Global, regional, and national burden of chronic kidney disease, 1990-2017: a systematic analysis for the Global Burden of Disease Study 2017. Lancet. 2020;395(10225):70933.

6. Ikizler TA, Robinson-Cohen C, Ellis C, Headley SAE, Tuttle K, Wood RJ, Evans EE, Milch CM, Moody KA, Germain $\mathrm{M}$, et al. Metabolic Effects of Diet and Exercise in Patients with Moderate to Severe CKD: A Randomized Clinical Trial. J Am Soc Nephrol. 2018;29(1):250-9.

7. Ajjarapu AS, Hinkle SN, Li M, Francis EC, Zhang C. Dietary Patterns and Renal Health Outcomes in the General Population: A Review Focusing on Prospective Studies. Nutrients 2019, 11(8).

8. Paterson EN, Neville CE, Silvestri G, Montgomery S, Moore E, Silvestri V, Cardwell CR, MacGillivray TJ, Maxwell AP, Woodside JV, et al. Dietary patterns and chronic kidney disease: a cross-sectional association in the Irish Nun Eye Study. Sci Rep. 2018;8(1):6654.

9. Abuyassin B, Sharma K, Ayas NT, Laher I. Obstructive Sleep Apnea and Kidney Disease: A Potential Bidirectional Relationship? J Clin Sleep Med. 2015;11(8):915-24.

10. Agyemang C, Snijder MB, Adjei DN, van den Born BJ, Modesti PA, Peters RJ, Stronks K, Vogt L. Ethnic Disparities in CKD in the Netherlands: The Healthy Life in an Urban Setting (HELIUS) Study. Am J Kidney Dis. 2016;67(3):391-9.

11. Nephrology JSo. Evidence-based Clinical Practice Guideline for CKD 2013. Clinical Experimental Nephrology. 2014;18(3):346-423.

12. Levey AS, Stevens LA, Schmid CH, Zhang YL, Castro AF 3rd, Feldman HI, Kusek JW, Eggers P, Van Lente $F$, Greene $T$, et al. A new equation to estimate glomerular filtration rate. Ann Intern Med. 2009;150(9):604-12.

13. Coresh J, Turin TC, Matsushita K, Sang Y, Ballew SH, Appel LJ, Arima H, Chadban SJ, Cirillo M, Djurdjev 0 , et al. Decline in estimated glomerular filtration rate and subsequent risk of end-stage renal disease and mortality. JAMA. 2014;311(24):2518-31.

14. Opoku S, Gan Y, Fu W, Chen D, Addo-Yobo E, Trofimovitch D, Yue W, Yan F, Wang Z, Lu Z. Prevalence and risk factors for dyslipidemia among adults in rural and urban China: findings from the China National Stroke Screening and prevention project (CNSSPP). BMC Public Health. 2019;19(1):1500-0.

15. Xu W, Zhang H, Paillard-Borg S, Zhu H, Qi X, Rizzuto D. Prevalence of Overweight and Obesity among Chinese Adults: Role of Adiposity Indicators and Age. Obes Facts. 2016;9(1):17-28. 
16. Guo H, Liu J, Zhang J, Ma R, Ding Y, Zhang M, He J, Xu S, Li S, Yan Y, et al. The Prevalence of Metabolic Syndrome Using Three Different Diagnostic Criteria among Low Earning Nomadic Kazakhs in the Far Northwest of China: New Cut-Off Points of Waist Circumference to Diagnose MetS and Its Implications. PLoS One. 2016;11(2):e0148976.

17. Joint committee issued Chinese guideline for the management of dyslipidemia in a. 2016 Chinese guideline for the management of dyslipidemia in adults. Zhonghua Xin Xue Guan Bing Za Zhi. 2016;44(10):833-53.

18. Huang X, Jiang X, Wang L, Chen L, Wu Y, Gao P, Hua F. Visceral adipose accumulation increased the risk of hyperuricemia among middle-aged and elderly adults: a population-based study. J Transl Med. 2019;17(1):341.

19. Wang SS, Lay S, Yu HN, Shen SR. Dietary Guidelines for Chinese Residents (2016): comments and comparisons. J Zhejiang Univ Sci B. 2016;17(9):649-56.

20. Anyzewska A, Lakomy R, Lepionka T, Szarska E, Maculewicz E, Tomczak A, Bertrandt J. Association Between Diet, Physical Activity and Body Mass Index, Fat Mass Index and Bone Mineral Density of Soldiers of the Polish Air Cavalry Units. Nutrients 2020, 12(1).

21. Rubin DB. Statistical Matching Using File Concatenation with Adjusted Weights and Multiple Imputations. Journal of Business Economic Statistics. 1986;4(1):87-94.

22. Akbarpour S, Khalili D, Zeraati H, Mansournia MA, Ramezankhani A, Fotouhi A. Healthy lifestyle behaviors and control of hypertension among adult hypertensive patients. Sci Rep. 2018;8(1):8508.

23. Barbaresko J, Rienks J, Nothlings U. Lifestyle Indices and Cardiovascular Disease Risk: A Metaanalysis. Am J Prev Med. 2018;55(4):555-64.

24. Lin CH, Chiang SL, Tzeng WC, Chiang LC. Systematic review of impact of lifestyle-modification programs on metabolic risks and patient-reported outcomes in adults with metabolic syndrome. Worldviews Evid Based Nurs. 2014;11(6):361-8.

25. Esposito K, Ciardiello F, Giugliano D. Unhealthy diets: a common soil for the association of metabolic syndrome and cancer. Endocrine. 2014;46(1):39-42.

26. Zhang N, Du SM, Ma GS. Current lifestyle factors that increase risk of T2DM in China. Eur J Clin Nutr. 2017;71(7):832-8.

27. Dunkler D, Kohl M, Teo KK, Heinze G, Dehghan M, Clase CM, Gao P, Yusuf S, Mann JF, Oberbauer R, et al. Population-Attributable Fractions of Modifiable Lifestyle Factors for CKD and Mortality in Individuals With Type 2 Diabetes: A Cohort Study. Am J Kidney Dis. 2016;68(1):29-40.

28. Luyckx VA, Tuttle KR, Garcia-Garcia G, Gharbi MB, Heerspink HJL, Johnson DW, Liu ZH, Massy ZA, Moe 0 , Nelson RG, et al. Reducing major risk factors for chronic kidney disease. Kidney Int Suppl (2011). 2017;7(2):71-87.

29. Fujibayashi K, Fukuda H, Yokokawa H, Haniu T, Oka F, Ooike M, Gunji T, Sasabe N, Okumura M, lijima K. Associations between Healthy Lifestyle Behaviors and Proteinuria and the Estimated Glomerular Filtration Rate (eGFR). Journal of Atherosclerosis Thrombosis. 2012;19(10):932-40\%।. 2020-20042005 2011:2053:2000. 
30. Michishita R, Matsuda T, Kawakami S, Tanaka S, Kiyonaga A, Tanaka H, Morito N, Higaki Y. The association between changes in lifestyle behaviors and the incidence of chronic kidney disease (CKD) in middle-aged and older men. J Epidemiol. 2017;27(8):389-97.

31. Michishita R, Matsuda T, Kawakami S, Kiyonaga A, Tanaka H, Morito N, Higaki Y. The accumulation of healthy lifestyle behaviors prevents the incidence of chronic kidney disease (CKD) in middle-aged and older males. Environ Health Prev Med. 2016;21(3):129-37.

32. Baylin A, Kabagambe EK, Ascherio A, Spiegelman D, Campos H. High 18:2 trans-fatty acids in adipose tissue are associated with increased risk of nonfatal acute myocardial infarction in costa rican adults. J Nutr. 2003;133(4):1186-91.

33. Kim SJ, Kwon SK, Kim HY, Kim SM, Bae JW, Choi JK. DPP-4 inhibition enhanced renal tubular and myocardial GLP-1 receptor expression decreased in CKD with myocardial infarction. BMC Nephrol. 2019;20(1):75.

34. Mesias M, Morales FJ. Effect of Different Flours on the Formation of Hydroxymethylfurfural, Furfural, and Dicarbonyl Compounds in Heated Glucose/Flour Systems. Foods 2017, 6(2).

35. Gill V, Kumar V, Singh K, Kumar A, Kim JJ. Advanced Glycation End Products (AGEs) May Be a Striking Link Between Modern Diet and Health. Biomolecules 2019, 9(12).

36. Totani N, Yawata M, Ojiri Y, Fujioka Y. Effects of trace acrylamide intake in Wistar rats. J Oleo Sci. 2007;56(9):501-6.

37. Abdel-Daim MM, Abd Eldaim MA, Hassan AG. Trigonella foenum-graecum ameliorates acrylamideinduced toxicity in rats: Roles of oxidative stress, proinflammatory cytokines, and DNA damage. Biochem Cell Biol. 2015;93(3):192-8.

38. Santin F, Canella D, Borges C, Lindholm B, Avesani CM. Dietary Patterns of Patients with Chronic Kidney Disease: The Influence of Treatment Modality. Nutrients 2019, 11(8).

39. Park I, Xun P, Tsinovoi CL, Klemmer P, Liu K, He K. Intakes of long-chain omega-3 polyunsaturated fatty acids and non-fried fish in relation to incidence of chronic kidney disease in young adults: a 25year follow-up. Eur J Nutr. 2020;59(1):399-407.

40. Haring B, Selvin E, Liang M, Coresh J, Grams ME, Petruski-lvleva N, Steffen LM, Rebholz CM. Dietary Protein Sources and Risk for Incident Chronic Kidney Disease: Results From the Atherosclerosis Risk in Communities (ARIC) Study. J Ren Nutr. 2017;27(4):233-42.

41. Yang XH, Zhang BL, Gu YH, Zhan XL, Guo LL, Jin HM. Association of sleep disorders, chronic pain, and fatigue with survival in patients with chronic kidney disease: a meta-analysis of clinical trials. Sleep Med. 2018;51:59-65.

42. Nam S, Whittemore R, Jung S, Latkin C, Kershaw T, Redeker NS. Physical neighborhood and social environment, beliefs about sleep, sleep hygiene behaviors, and sleep quality among African Americans. Sleep Health. 2018;4(3):258-64.

43. Hassanzadeh-Taheri M, Hassanzadeh-Taheri M, Jahani F, Erfanian Z, Moodi H, Hosseini M. The impact of long-term consumption of diets enriched with olive, cottonseed or sesame oils on kidney morphology: A stereological study. An Acad Bras Cienc. 2019;91(2):e20180855. 
44. Szczuko M, Kaczkan M, Drozd A, Maciejewska D, Palma J, Owczarzak A, Marczuk N, Rutkowski P, Malgorzewicz S. Comparison of Fatty Acid Profiles in a Group of Female Patients with Chronic Kidney Diseases (CKD) and Metabolic Syndrome (MetS)(-)Similar Trends of Changes, Different Pathophysiology. Int J Mol Sci. 2019;20(7):1719.

45. Sijtsma FP, Meyer KA, Steffen LM, Shikany JM, Van Horn L, Harnack L, Kromhout D, Jacobs DR Jr. Longitudinal trends in diet and effects of sex, race, and education on dietary quality score change: the Coronary Artery Risk Development in Young Adults study. Am J Clin Nutr. 2012;95(3):580-6.

\section{Figures}

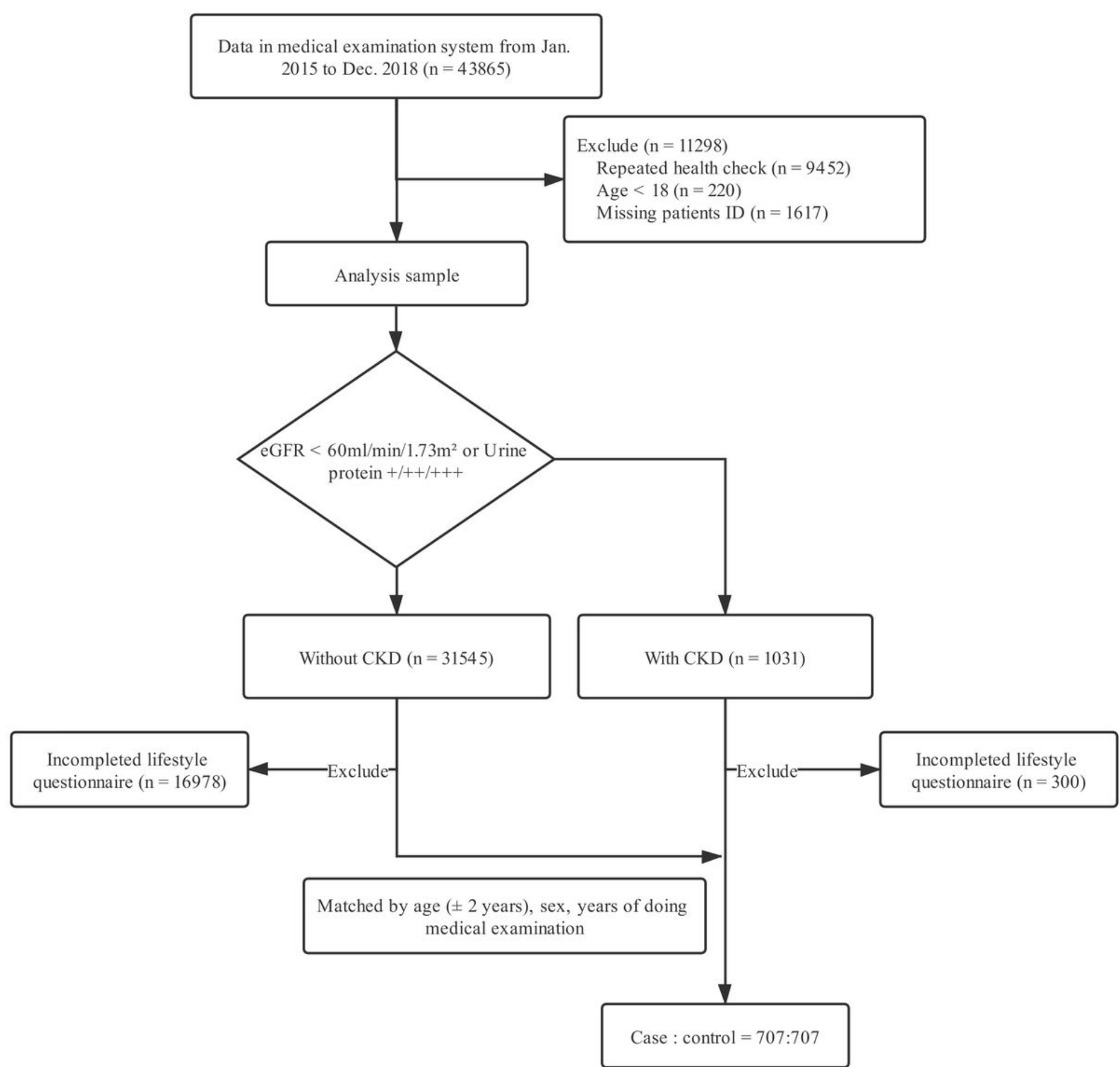


Figure 1

Flowchart for the case control study design. CKD: chronic kidney disease; eGFR: estimated glomerular filtration rate.

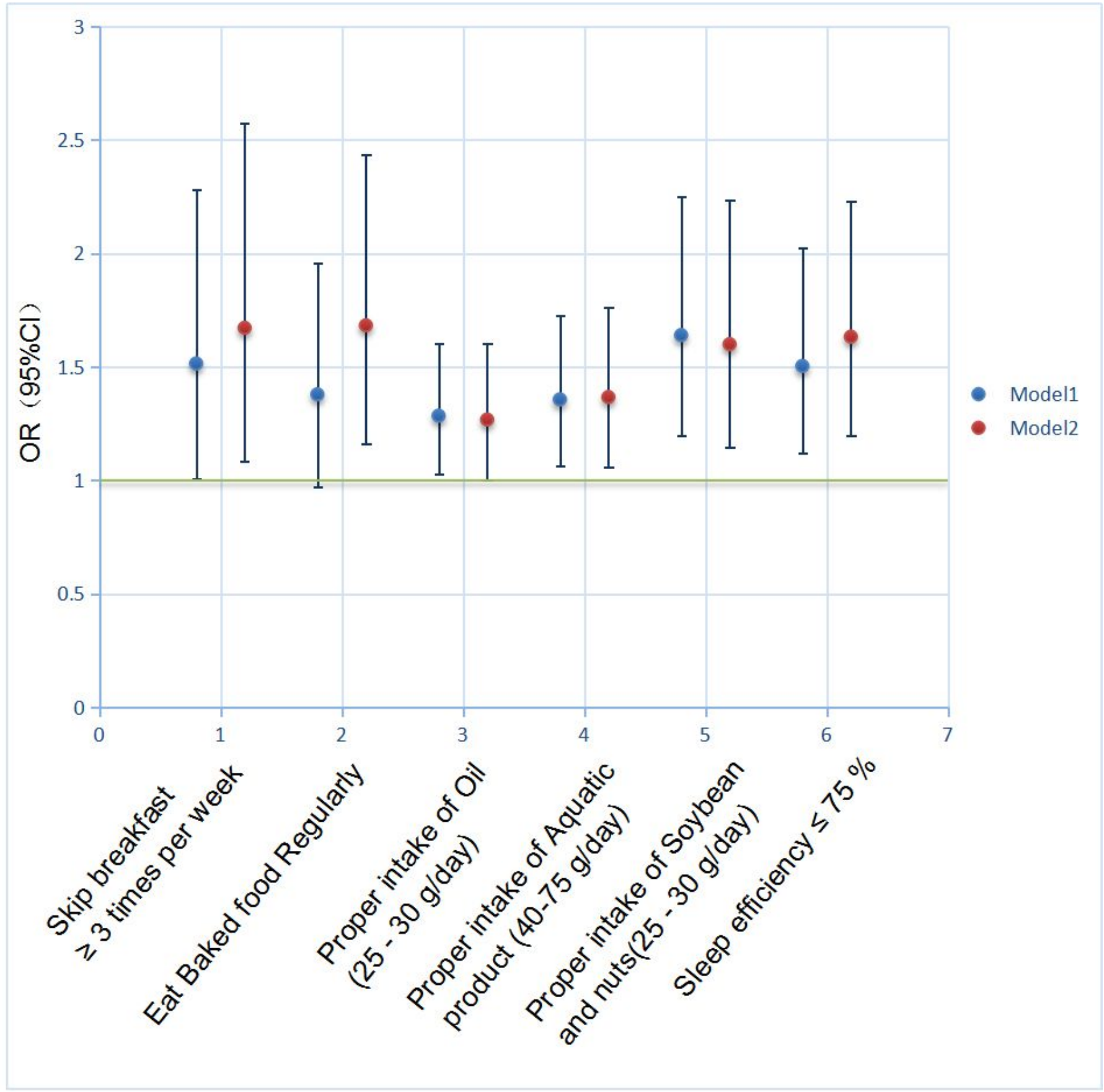

Figure 2

ORs of CKD and corresponding $95 \%$ Cls according to different variables. Data are expressed as odds ratio (95\% confidence interval [CI]). BMI: Body mass index; WC: Waist circumference; DBP: Diastolic 
blood pressure; SBP: Systolic blood pressure; TG: Triglycerides; HDL-C: High-Density Lipoprotein Cholesterol; VLDL-C: Very Low-Density Lipoprotein Cholesterol; SUA: Serum uric acid; FBG: Fasting Blood Glucose. Model 1: adjustment for age, sex; Model 2: adjustment for age, sex, weight, BMI, WC, DBP, SBP, TG, HDL-C, VLDL-C, SUA, FBG, hyperuricemia, metabolic syndrome, WC groups. 